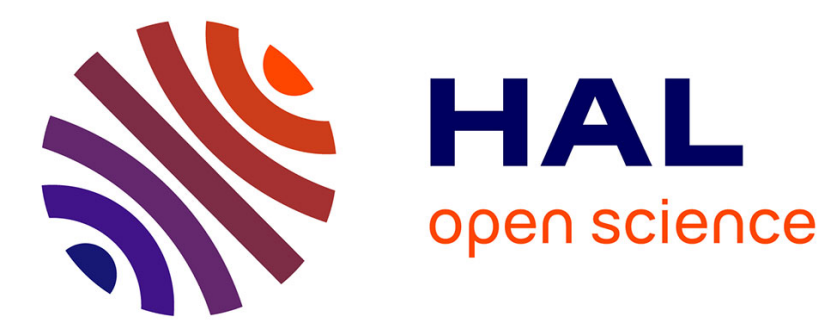

\title{
The marginal propensity to consume and multidimensional risk
}

Elyès Jouini, Clotilde Napp, Diego Nocetti

\section{To cite this version:}

Elyès Jouini, Clotilde Napp, Diego Nocetti. The marginal propensity to consume and multidimensional risk. Economics Letters, 2013, 119 (2), pp.124-127. halshs-00927262

\section{HAL Id: halshs-00927262 https://shs.hal.science/halshs-00927262}

Submitted on 12 Jan 2014

HAL is a multi-disciplinary open access archive for the deposit and dissemination of scientific research documents, whether they are published or not. The documents may come from teaching and research institutions in France or abroad, or from public or private research centers.
L'archive ouverte pluridisciplinaire HAL, est destinée au dépôt et à la diffusion de documents scientifiques de niveau recherche, publiés ou non, émanant des établissements d'enseignement et de recherche français ou étrangers, des laboratoires publics ou privés. 


\title{
The Marginal Propensity to Consume and Multidimensional Risk
}

\author{
Elyès Jouini \\ Ceremade
}

\author{
Clotilde Napp \\ CNRS-DRM
}

\author{
Diego Nocetti \\ Clarkson University
}

January 14, 2013

\begin{abstract}
Kimball (1990) established that income risk increases the marginal propensity to consume if and only if absolute prudence is decreasing. We characterize decreasing and increasing multivariate prudence and we show that a multidimensional risk increases the marginal propensity to consume if and only if a matrix-measure of multivariate prudence decreases with wealth, in the sense that its derivative is negative-definite.
\end{abstract}

\section{Introduction}

A substantial amount of research has been devoted to understanding the effect of uncertainty on consumption and saving decisions. In a numerical exercise, Zeldes (1989) showed that the presence of uncertainty has a strong positive effect on the level and on the slope of the consumption function (i.e. the marginal propensity to consume (MPC)). Kimball (1990a,b) established formally the necessary and sufficient condition for the introduction of uncertainty to increase the MPC. He showed that consumption exhibits "excess sensitivity" in the presence of risk if prudence is decreasing in income, where prudence is measured by the index $p(x) \equiv \frac{-v^{\prime \prime \prime}(x)}{v^{\prime \prime}(x)}$. Therefore, while $p(x)$ measures the strength of the precautionary saving motive, $p^{\prime}(x)$ captures the response of the MPC to risk ${ }^{1}$.

Kimball's result pertains to the case in which utility is a function of a single attribute, income. Yet, most consumers face multiple sources of risk. For example, a consumer may select his saving and consumption without having full knowledge of future prices or his future health status. While a number of recent papers have evaluated precautionary saving motives in the presence of multiple risks (e.g. Courbage and Rey (2007), Menegatti (2009), Denuit, Eeckhoudt, and Menegatti (2011)), the effect of a multidimensional risk on the marginal propensity to consume has not been established. The objective of this note is to fill this gap in the literature.

Our analysis complements the results of Jouini, Napp, and Nocetti (2012) -JNN-, who characterize comparative multivariate prudence by making use of a matrix-measure $P(x)$

\footnotetext{
${ }^{1}$ Carroll and Kimball (1996) later established conditions for uncertainty to induce a concave consumption function (i.e. for the MPC to decrease with wealth).
} 
that captures the intensity of the precautionary saving motive. We use $P(x)$ to define decreasing, constant, and increasing multivariate prudence, and we show that the MPC is higher in the presence of a multidimensional risk if and only if $P(x)$ is decreasing in wealth $x_{0}$, in the sense that $-\frac{\partial}{\partial x_{0}} P^{w}(x)$ is positive-definite.

\section{Preliminaries on multivariate prudence}

We begin by revisiting the model and the results of JNN. The consumer lives two periods, derives utility from $(n+1)$ attributes, and is endowed with $(n+1)$-dimensional, increasing and concave first and second period utility functions $u$ and $v$. The first attribute is the income. Many interpretations are possible for the other variables, including a vector of market prices, non-traded commodities (e.g. health status), or social attributes (e.g. social recognition). We let $y=\left(y_{0}, \ldots, y_{n}\right)$ and $x=\left(x_{0}, \ldots, x_{n}\right)$ denote the initial endowments of the individual in the $(n+1)$ attributes respectively at the first and second period.

In the first period, the individual saves an amount $s$ of income. Assuming that current monetary investments only have monetary consequences, this saving enables the individual to obtain $\rho_{0} s$ of income at the second period ${ }^{2}$. For simplicity of notation, we introduce the function $w \equiv \frac{\partial v}{\partial x_{0}} \rho_{0}$, which represents the second period marginal utility of saving, and we also introduce the vector $\delta \equiv(1,0, \ldots, 0)$.

Under certainty, the consumption/saving problem is

$$
\max _{s \in \mathbb{R}} h(s)=u\left(y_{0}-s, y_{1}, \ldots, y_{n}\right)+v\left(x_{0}+\rho_{0} s, x_{1}, \ldots, x_{n}\right) .
$$

The solution $s^{*}$ satisfies $h^{\prime}\left(s^{*}\right)=-u_{0}\left(y-s^{*} \delta\right)+w\left(x+s^{*} \rho_{0} \delta\right)=0$.

Consider now the case with multivariate risk. There is noise $\widetilde{e}=\left(\widetilde{e}_{0}, \ldots, \widetilde{e}_{n}\right)$ affecting the vector $x$ of second period consumption, where $E[\widetilde{e}]=0$. We denote by $\widetilde{x} \equiv x+\widetilde{e}$ the vector of second period noisy consumption and by $V^{e} \equiv\left[\sigma_{i j}\right]$ with $\sigma_{i j}=\operatorname{cov}\left(\widetilde{e}_{i}, \widetilde{e}_{j}\right)$, the $(n+1) \times(n+1)$ variance-covariance matrix of $\widetilde{e}$. The consumption/saving problem becomes

$$
\max _{s \in \mathbb{R}} H(s)=u\left(y_{0}-s, y_{1}, \ldots, y_{n}\right)+E\left[v\left(\widetilde{x}_{0}+\rho_{0} s, \widetilde{x}_{1}, \ldots, \widetilde{x}_{n}\right)\right]
$$

The solution is denoted by $\widehat{s}$ and is characterized by $H^{\prime}(\widehat{s})=u_{0}(y-\widehat{s} \delta)=E\left[w\left(\widetilde{x}+\widehat{s} \rho_{0} \delta\right)\right]$.

An individual is multivariate prudent if $\widehat{s} \geq s^{*}$ for all $(z, x)$. Equivalently, an individual is multivariate prudent if the equivalent precautionary premium $\Psi(x, \widetilde{e}, w)$ is non-negative, where $\Psi(x, \widetilde{e}, w)$ is defined by

$$
E[w(\widetilde{x})]=w(x-\Psi(x, \widetilde{e}, w) \delta) .
$$

It corresponds to the certain reduction of second period income that has the same upward effect on the optimal level of first period saving as the introduction of the additional risk.

JNN propose $P^{w} \equiv\left[-\frac{w_{i j}}{w_{0}}\right]$ as a matrix-measure of local multivariate prudence and show that it unambiguously captures the intensity of the precautionary saving motive in a

\footnotetext{
${ }^{2}$ JNN evaluate a more general model in which current monetary investments have multidimensional consequences in the second period. In this paper, we consider the more standard model (e.g. Courbage and Rey (2007), Menegatti (2009), Denuit et al. (2011)) in which second period consequences are unidimensional.
} 
multivariate setting for both small and large multidimensional risks. Indeed, they obtain that the precautionary premium is nonnegative (i.e. $\Psi(x, \widetilde{e}, w) \geq 0$, for all $(x, \widetilde{e})$ ) if and only if the matrix-measure of multivariate prudence $P^{w}(x)$ is positive semidefinite for all $x$. When comparing two agents, they obtain that Agent A is more prudent than Agent $B$, (i.e., $\Psi\left(x, \widetilde{e}, w_{A}\right) \geq \Psi\left(x, \widetilde{e}, w_{B}\right)$ for all $\left.(x, \widetilde{e})\right)$ if and only if $\left(P^{w_{A}}-P^{w_{B}}\right)(x)$ is positive semidefinite for all $x$.

\section{Decreasing, constant, and increasing multivariate pru- dence in the $j^{\text {th }}$ attribute}

Pratt (1964) established the intuitive fact that decreasing absolute risk aversion implies that the risk premium is decreasing in wealth. Kimball (1990b) mapped this result to the case of precautionary saving, establishing that decreasing absolute prudence, as measured by the univariate function that he proposed, implies that the precautionary saving premium is decreasing in wealth.

We obtain the following result

Proposition 1 The following conditions are equivalent:

1. The precautionary premium is a decreasing (resp. constant, increasing) function in attribute j, i.e. $\frac{\partial \Psi(x, \widetilde{e}, w)}{\partial x_{j}}<0$ (resp. $\left.\frac{\partial \Psi(x, \widetilde{e}, w)}{\partial x_{j}}=0, \frac{\partial \Psi(x, \widetilde{e}, w)}{\partial x_{j}}>0\right)$.

2. The matrix $-\frac{\partial}{\partial x_{j}} P^{w}(x)$ is positive-definite (resp. null, negative-definite).

Proof The precautionary premium is a decreasing function of $x_{j}$ if and only if we have $\Psi(x, \widetilde{e}, w) \geq \Psi(x+d, \widetilde{e}, w)$ for all $d \quad \in \quad D_{j}$ with $D_{j}=\left\{\left(d_{0}, \cdots, d_{n}\right): d_{i}=0, i \neq j\right.$, and $\left.d_{j}>0\right\}$. Let us introduce $v_{d}$ the utility function defined by $v_{d}(x)=v(x+d)$ and let us define $w_{d}$ by $w_{d}\left(x_{0}, x_{1}, \ldots, x_{n}\right)=\rho_{0} \frac{\partial v_{d}}{\partial x_{0}}\left(x_{0}, x_{1}, \ldots, x_{n}\right)$. We clearly have $w_{d}(x)=w(x+d)$ and we check that $\Psi(x+d, \widetilde{e}, w)=\Psi\left(x, \widetilde{e}, w_{d}\right)$. We have then the following characterization. The precautionary premium is a decreasing function of $x_{j}$ if and only if we have $\Psi(x, \widetilde{e}, w) \geq \Psi\left(x, \widetilde{e}, w_{d}\right)$ for all $d \in D_{j}$. From Proposition 3 in JNN, this is equivalent to the statement that $P^{w}(x)-P^{w_{d}}(x)$ is positive semi-definite for all $d \in D_{j}$. Remark that $P^{w_{d}}(x)=P^{w}(x+d)$ and that $P^{w}(x)-P^{w}(x+d)$ is positive semi-definite for all $d \in D_{j}$ if and only if $-\frac{\partial}{\partial x_{j}} P^{w}(x)$ is positive semi-definite.

We retrieve the fact that the precautionary saving premium is decreasing (with respect to attribute $j$ ) if and only if the matrix measure of multivariate prudence is decreasing (with respect to attribute $j$ ) in the sense that its derivative (with respect to attribute $j$ ) is negative-definite.

\section{Multivariate prudence and the MPC}

We are now ready to present our main result. The consumption function is defined by

$$
c(x, \tilde{e})=\arg \max u\left(c, y_{1}, \ldots, y_{n}\right)+E\left[v\left(\tilde{x}_{0}+\rho_{0}\left(y_{0}-c\right), \tilde{x}_{1}, \ldots, \tilde{x}_{n}\right)\right]
$$


where $\left(y_{0}, y_{1}, \ldots, y_{n}\right)$ is kept fixed. The marginal propensity to consume out of wealth is given by $\frac{\partial c}{\partial x_{0}}$ and we want to compare $\frac{\partial c}{\partial x_{0}}(x, \tilde{e})$ with $\frac{\partial c}{\partial x_{0}}(x, 0)$; that is to say, we want to analyze the impact of the multidimensional risk $\tilde{e}$ on the marginal propensity to consume out of wealth. In the next, the inverse of $c$ (more precisely, the inverse of the function $x_{0} \rightarrow c(x, \tilde{e})$ ) is denoted by $g\left(c ; x_{1}, \ldots, x_{n}, \tilde{e}_{0}, \ldots, \tilde{e}_{n}\right)$. By definition, it satisfies

$$
g\left(c(x, \tilde{e}) ; x_{1}, \ldots, x_{n}, \tilde{e}_{0}, \ldots, \tilde{e}_{n}\right)=x_{0} .
$$

For a given function $w$ and a given $\tilde{X}$, we also define the compensating precautionary premium $\Psi^{*}(X, \widetilde{e}, w)$ by

$$
w\left(x_{0}, \ldots, x_{n}\right)=E\left[w\left(\tilde{x}_{0}+\Psi^{*}(x, \widetilde{e}, w), \tilde{x}_{1}, \ldots, \tilde{x}_{n}\right)\right] .
$$

The compensating precautionary premium is the additional amount of income that induces the consumer to save the same amount in the presence of the multidimensional risk $\tilde{e}$ as in the absence of it. The following Proposition establishes the link between changes in the equivalent and compensating precautionary premia and the effect of the multidimensional risk on the MPC.

Proposition 2 The following conditions are equivalent

1. The equivalent precautionary premium $\Psi(x, \widetilde{e}, w)$ is decreasing (resp. constant, increasing) with respect to $x_{0}$.

2. The compensating precautionary premium $\Psi^{*}(x, \widetilde{e}, w)$ is decreasing (resp. constant, increasing) with respect to $x_{0}$.

3. The marginal propensity to consume out of wealth is higher (resp. the same, lower) in the presence of the multidimensional risk $\tilde{e}$.

Proof $(1) \Leftrightarrow(2)$ : When $X$ and $\widetilde{e}$ are given and when $\Psi(x, \widetilde{e}, w)$ is decreasing in $x_{0}$, the function $x_{0} \rightarrow x_{0}-\Psi(x, \widetilde{e}, w)$ is increasing and we denote by $f\left(x_{0} ; x_{1}, \ldots, x_{n}, \widetilde{e}_{0}, \ldots, \widetilde{e}_{n}\right)$ its inverse. By definition of $\Psi(x, \widetilde{e}, w)$ we have then

$$
w(X)=E\left[w\left(f\left(x_{0} ; x_{1}, \ldots, x_{n}, \widetilde{e}_{0}, \ldots, \widetilde{e}_{n}\right)+\widetilde{e}_{0}, \tilde{x}_{1}, \ldots, \tilde{x}_{n}\right)\right]
$$

and we have then that $f\left(x_{0} ; x_{1}, \ldots, x_{n}, \widetilde{e}_{0}, \ldots, \widetilde{e}_{n}\right)=x_{0}+\Psi^{*}(x, \widetilde{e}, w)$. Since $\Psi(x, \widetilde{e}, w)$ is decreasing in $x_{0}, x_{0}-\Psi(x, \widetilde{e}, w)$ increases faster than $x_{0}$ and $f\left(x_{0} ; x_{1}, \ldots, x_{n}, \widetilde{e}_{0}, \ldots, \widetilde{e}_{n}\right)$ increases in $x_{0}$ at a slower rate than $x_{0}$ which gives that $\Psi^{*}(x, \widetilde{e}, w)$ decreases in $x_{0}$. It is easy to check that a similar argument gives that $\Psi^{*}(x, \widetilde{e}, w)$ increases in $x_{0}$ when $\Psi(x, \widetilde{e}, w)$ increases in $x_{0}$.

$(2) \Leftrightarrow(3)$ : By definition of $g$, we have

$$
\frac{\partial u}{\partial y_{0}}\left(c, y_{1}, \ldots, y_{n}\right)=E\left[w\left(g\left(c ; x_{1}, \ldots, x_{n}, \widetilde{e}_{0}, \ldots, \widetilde{e}_{n}\right)+\widetilde{e}_{0}+\rho_{0}\left(y_{0}-c\right), \tilde{x}_{1}, \ldots, \tilde{x}_{n}\right)\right]
$$

and since the left term does not depend on $\tilde{e}$ we have

$$
\frac{\partial u}{\partial y_{0}}\left(c, y_{1}, \ldots, y_{n}\right)=w\left(g\left(c ; x_{1}, \ldots, x_{n}, 0, \ldots, 0\right)+\rho_{0}\left(y_{0}-c\right), x_{1}, \ldots, x_{n}\right)
$$


By definition of $\Psi^{*}(x, \widetilde{e}, w)$ we have

$$
\begin{aligned}
& w\left(g\left(c ; x_{1}, \ldots, x_{n}, 0, \ldots, 0\right)+\rho_{0}\left(y_{0}-c\right), x_{1}, \ldots, x_{n}\right) \\
= & E\left[w\left(g\left(c ; x_{1}, \ldots, x_{n}, 0, \ldots, 0\right)+\rho_{0}\left(y_{0}-c\right)+\widetilde{e}_{0}+\Psi^{*}(x, \widetilde{e}, w), \tilde{x}_{1}, \ldots, \tilde{x}_{n}\right)\right]
\end{aligned}
$$

with $x=\left(g\left(c ; x_{1}, \ldots, x_{n}, 0, \ldots, 0\right)+\rho_{0}\left(y_{0}-c\right), x_{1}, \ldots, x_{n}\right)$.

We have then

$$
\begin{aligned}
& E\left[w\left(g\left(c ; x_{1}, \ldots, x_{n}, e_{0}, \ldots, e_{n}\right)+\widetilde{e}_{0}+\rho_{0}\left(y_{0}-c\right), \tilde{x}_{1}, \ldots, \tilde{x}_{n}\right)\right] \\
= & E\left[w\left(g\left(c ; x_{1}, \ldots, x_{n}, 0, \ldots, 0\right)+\widetilde{e}_{0}+\rho_{0}\left(y_{0}-c\right)+\Psi^{*}(x, \widetilde{e}, w), \tilde{x}_{1}, \ldots, \tilde{x}_{n}\right)\right]
\end{aligned}
$$

and since $w$ is decreasing in $x_{0}$ this gives

$$
g\left(c ; x_{1}, \ldots, x_{n}, 0, \ldots, 0\right)+\Psi^{*}(x, \widetilde{e}, w)=g\left(c ; x_{1}, \ldots, x_{n}, \widetilde{e}_{0}, \ldots, \widetilde{e}_{n}\right) .
$$

Differentiating this equation with respect to $c$ gives

$$
\begin{aligned}
& \frac{\partial g}{\partial c}\left(c ; x_{1}, \ldots, x_{n}, \widetilde{e}_{0}, \ldots, \widetilde{e}_{n}\right) \\
= & \frac{\partial g}{\partial c}\left(c ; x_{1}, \ldots, x_{n}, 0, \ldots, 0\right) \\
& +\left(\frac{\partial g}{\partial c}\left(c ; x_{1}, \ldots, x_{n}, 0, \ldots, 0\right)-\rho_{0}\right) \frac{\partial \Psi^{*}}{\partial x_{0}}(x, \widetilde{e}, w) .
\end{aligned}
$$

Differentiating Equation (4) with respect to $y_{0}$ gives

$$
\frac{\partial^{2} u}{\partial y_{0}^{2}}\left(c, y_{1}, \ldots, y_{n}\right)=\left(\frac{\partial g}{\partial c}\left(c ; x_{1}, \ldots, x_{n}, 0, \ldots, 0\right)-\rho_{0}\right) E\left[\frac{\partial w}{\partial x_{0}}(\widetilde{Z})\right]
$$

with $\widetilde{Z}=\left(g\left(c ; x_{1}, \ldots, x_{n}, \widetilde{e}_{0}, \ldots, \widetilde{e}_{n}\right)+\widetilde{e}_{0}+\rho_{0}\left(y_{0}-c\right), \tilde{x}_{1}, \ldots, \tilde{x}_{n}\right)$.

Since $u$ is concave and $w$ decreasing, we have $\frac{\partial g}{\partial c}\left(c ; x_{1}, \ldots, x_{n}, 0, \ldots, 0\right)-\rho_{0}>0$. If $\Psi^{*}(x, \widetilde{e}, w)$ is decreasing in $x_{0}$, Equation (5) gives us that

$$
\frac{\partial g}{\partial c}\left(c ; x_{1}, \ldots, x_{n}, \widetilde{e}_{0}, \ldots, \widetilde{e}_{n}\right)<\frac{\partial g}{\partial c}\left(c ; x_{1}, \ldots, x_{n}, 0, \ldots, 0\right) .
$$

The impact of the multidimensional risk is then towards a decrease of $\frac{\partial g}{\partial c}$. Since $g$ is the inverse of the consumption function, this means that the impact of the multidimensional risk is towards an increase of the marginal propensity to consume out of wealth. We have the opposite impact when $\Psi^{*}(x, \widetilde{e}, w)$ is increasing in $x_{0}$.

Linking Proposition 2 with Proposition 1 we obtain that a necessary and sufficient condition for the MPC to be higher in the presence of the multidimensional risk $\tilde{e}$ is that the matrix measure of multivariate prudence is decreasing in wealth, in the sense that $-\frac{\partial}{\partial x_{0}} P^{w}(x)$ is positive-definite. Importantly, this condition implies that the decrease of the usual measure of prudence, $\frac{-v_{000}}{v_{00}}$, is generally neither sufficient nor necessary to establish whether the MPC is higher or lower in the presence of a risk that is multidimensional. Instead, the necessary and sufficient condition requires information about preferences towards all the attributes that enter the utility function. 


\section{An Example}

To illustrate our results, imagine a world with two tradable goods $a$ and $b$. Suppose that in each of two dates the consumer selects how much to purchase of each good and in the first period the consumer also selects how much to save $s$ out of his or her date 0 income $z_{0}$ (in terms of the first good). The date 1 income in terms of good $a$ is denoted by $z_{1}$. Let $a_{i}$ and $b_{i}, i=0,1$, be the amount consumed of the goods at date $i$, and let $q_{i}$ be the relative price of good $b$ at date $i$ in terms of good $a$. Then, the budget constraint for date 0 is $a_{0}+q_{0} b_{0}=z_{0}-s$ and the corresponding constraint for date 1 is $a_{1}+q_{1} b_{1}=z_{1}+s$. We assume that there is uncertainty over the date 1 income and the date 1 relative price. In particular, we assume $\widetilde{z}_{1}=z_{0}+\widetilde{e}_{z}$ and $\widetilde{q}_{1}=q_{0}+\widetilde{e}_{q}$, where $\widetilde{e}_{z}$ and $\widetilde{e}_{q}$ are mean-zero random variables with a variance-covariance matrix $V^{e} \equiv\left[\sigma_{i j}\right]$ with $\sigma_{i j}=\operatorname{cov}\left(\widetilde{e}_{z}, \widetilde{e}_{q}\right)$. We also assume that the date 1 allocation between the two goods is done after the realization of the random shocks. Finally, we assume that the period $i$ utility has a power/Cobb-Douglas form: $u\left(a_{i}, b_{i}\right)=\frac{\left(a_{i}^{\alpha} b_{i}^{\beta}\right)^{1-\gamma}}{1-\gamma}$, with $\alpha+\beta=1 .^{3}$

To evaluate the effect of the multidimensional risk on saving behavior we can proceed in two steps:

1) Choose $a_{i}$ and $b_{i}$ to maximize utility at each date, the level $s$ of saving being given. This yields the lifetime indirect utility function

$$
H(s)=K\left\{\left[q_{0}^{\beta}\left(z_{0}-s\right)\right]^{1-\gamma}+E\left[q_{1}^{\beta}\left(z_{1}+s\right)\right]^{1-\gamma}\right\}
$$

where $K$ is a constant.

2) Select savings to maximize the indirect utility function. The first order condition for this problem is

$$
q_{0}^{\beta(1-\gamma)}\left(z_{0}-s\right)^{-\gamma}=E\left[q_{1}^{\beta(1-\gamma)}\left(z_{1}+s\right)^{-\gamma}\right] .
$$

Clearly, this is an example of our more general model with $y=\left(z_{0}, q_{0}\right)$ and $x=$ $\left(z_{1}, q_{1}\right)$. We readily retrieve that the local compensating precautionary premium equals $\Psi=\frac{1}{2} \operatorname{tr}\left[V^{e} P\right]$, where the matrix measure of multivariate prudence $P$ is given by

$$
P=\left[\begin{array}{cc}
(1+\gamma) z_{0}^{-1} & \beta(1-\gamma) q_{0}^{-1} \\
\beta(1-\gamma) q_{0}^{-1} & \beta(1-\gamma)[1+\beta(1-\gamma)] \gamma^{-1} z_{0} q_{0}^{-2}
\end{array}\right]
$$

As a consequence, a positive third derivative of the utility function with respect to income is not sufficient for a positive precautionary saving motive. Instead, a sufficient condition for $P$ to be positive semi-definite for all $z$ and $q$, and as a result for multivariate prudence to occur, is $\gamma \leq 1$. Furthermore, the precautionary premium is decreasing in income, and so $-\frac{\partial}{\partial x_{0}} P$ is positive definite and the MPC is higher in the presence of the multidimensional risk, if $1 \leq \gamma \leq \frac{1+\beta}{\beta}$. This contrasts sharply with the classical univariate model in which, given isoelastic preferences, the precautionary premium is unambiguously positive and decreasing in income.

\footnotetext{
${ }^{3}$ The case of labor income uncertainty with endogenous labor supply is a sub-case of this example in which $q=z$ is the wage rate, $b$ is leisure demand, and $a$ consumption (See Floden, 2006, and Nocetti and Smith, 2011).
} 


\section{References}

[1] Carroll C., Kimball, M. (1996) On the concavity of the consumption function. Econometrica 64, 981-992.

[2] Courbage C., Rey B. (2007) Precautionary saving in the presence of other risks. Economic Theory $32,417-424$.

[3] Denuit, M., Eeckhoudt, L., Menegatti, M. (2011) Correlated Risks, Bivariate Utility and Optimal Choices. Economic Theory 46, 39-54.

[4] Nocetti D., Smith, W.T. (2011). Precautionary saving and endogenous labor supply with and without intertemporal expected utility. Journal of Money, Credit, and Banking 43, 1475-1504.

[5] Floden, M. (2006). Labor supply and saving under uncertainty. Economic Journal 116, 721-737.

[6] Jouini, E., Napp, C., Nocetti, D. (2012) On Multivariate Prudence. Journal of Economic Theory (To appear).

[7] Kimball, M. (1990a). Precautionary saving and the marginal propensity to consume. NBER working paper 3403

[8] Kimball, M. (1990b) Precautionary saving in the small and in the large. Econometrica, $58,58-73$.

[9] Menegatti, M. (2009). Precautionary saving in the presence of other risks: A comment. Economic Theory 39, 473-476.

[10] Pratt, J.W. (1964) Risk aversion in the small and in the large. Econometrica 32, 122-136.

[11] Zeldes, S. (1989) Optimal Consumption with Stochastic Income: Deviations from Certainty Equivalence. Quarterly Journal of Economics 104, 275-298. 